\title{
An Expert CAD System for the Design of Marine Power Plants Using Artificial Intelligence*
}

\author{
Shinsuke AKAGI**, Toshiyuki TANAKA*** \\ and Hidetoshi KUBONISHI**
}

\begin{abstract}
An expert CAD system is developed for the design of marine power plants using the Artificial Intelligence (AI) concept. Firstly, the design process is discussed generally from the view point of applying the AI technique effectively to the design process. It is found that a hybrid-type expert CAD system coupled with the AI technique and the design optimization method is most effective for this type of CAD system. The system architecture of the devoloped CAD system consists of the knowledge base for the design rules, the frame-type data base of the plant machinery, and the mathematical optimization process. Through system execution, it is ascertained that the system is effective not only as a tool for plant design but also as a tool for instructing inexperienced designers.
\end{abstract}

Key Words : Desigin Engineering, CAD, Artificial Intelligence, Knowledge Engineer. ing, Expert System, Marine Power Plant

\section{Introduction}

The introduction of AI (Artificial Intelligence) technology, particularly in the form of an expert system, has recently drawn much attention as a powerful tool for CAD systems ${ }^{(1) \sim(4)}$. AI technology is expected to expand the applications of computers to engineering design beyond the conventional domain, which is still restricted to applications for engineering drawing.

However, this radically new technology is just starting. It is not yet known how to apply the technique effectively to engineering design. Investigations are required not only to explore the AI technology but also to understand the characteristics of the process of engineering design.

In this study, firstly the characteristics of the

*Received 27th June, 1987. Paper No. 86-0307 B, 86$1001 \mathrm{~A}$

**Department of Mechanical Engineering, Osaka University, 2-1 Yamadaoka, Suita, Osaka, 565, Japan

***Toyota Motor Corporation, 1 Toyotacho, Toyota, Aichi, 471, Japan engineering design process are discussed generally from the view point of the application of AI technology, and then an expert system for the design of marine power plants will be developed to ascertain its validity and effectiveness.

In the course of the development of the system, the concept of combining the AI technique and the numerical optimization method is adopted to solve the problem of design synthesis. This concept is based on the idea that some part of the domain of the design processes whose solutions depend on designers' experiences, i. e., knowledge of design, is supported by the AI technique, and the other domain is solved by the - mathematical optimization method.

2. Understanding the Engineering Design Process and Its Supporting Methodologies

The design process is characterized by a sequence of searching the design candidates, and decisionmaking to select the best design solution which satisfies design objectives or various required functions.

Figure 1 illustrates the typical design process and its supporting methodologies. The design process can 
be divided into several steps as shown in Fig. 1, namely ;

(1) Recognition of design needs,

(2) Search of the design solutions satisfying the design needs, i. e., design candidates or feasible solutions,

(3) Evaluation of the "acceptable" design solutions among the candidates. The standard for what is "acceptable" may be lower than that for the optimum, and the acceptability criteria are derived from the knowledge of design, i. e., the rules, the reliability, the geometric compatibility, and the time and money goals etc ${ }^{(1)}$.

(4) Determination of the design variable optimizing the design objectives. The design objectives here are usually limited to the criteria which can be expressed quantitatively among the criteria defined in the above item ( 3 ).

The characteristics of the above items indicate that the categories of the design process change from the ill-defined domain ${ }^{(1)}$ to the well-defined domain as the design process advances from item (1) to (4). The design methodologies to support the above process are also illustrated in Fig. 1.

The method of supporting the item (1) in the figure seems still very poor today, although some modelling methods such as the ISM (Interpretative Structural Modelling) technique have been found ${ }^{(5)}$.

For items (2) and (3) in Fig. 1, these steps are characterized by the fact that the design candidates are searched and evaluated by the "design knowl-

Design Process

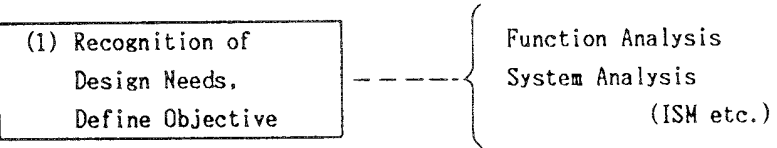

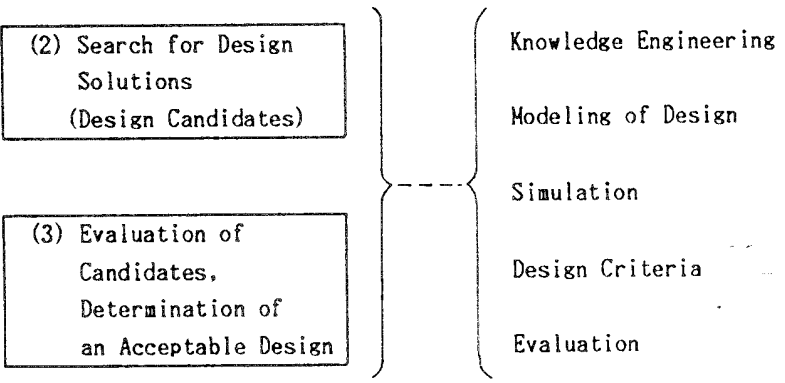

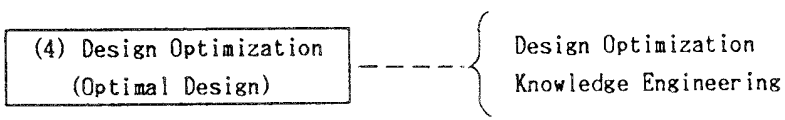

Fig. 1 Design process and the supporting design methodology edge". Current AI strategies are applicable to solve this kind of ill-defined problem.

Finally, the design step of item (4) can be supported by the usual mathematical optimization method.

\section{Basic Concept of Expert CAD Systems}

In this investigation, an expert CAD system will be developed to support a designer interactively and flexibly based on the results of the above discussion by adopting the AI technique into steps (2) and (3) in Fig. 1, coupling with the mathematical optimization method for step (4) in the figure. The concept is schematically shown in Fig. 2.

The system consists of two-level processes corresponding to the $\mathrm{AI}$ and the mathematical optimization procedure which can support a designer by making the best use of the merits of both techniques. In step 1, the design candidates are selected using the knowledge for design; the candidates here mean the acceptable type or configuration of the system components. In step 2, the optimization is performed for the design candidate selected in step 1; the optimization here means to determine the optimal size, capacity etc. for the selected candidate.

An expert system will be developed here for the design of a marine power plant to verify the above concept.

\section{Outline of a Marine Power Plant and Its Design Procedure}

Before developing the system, the basic structure of a marine power plant and its design procedure are introduced briefly.

Figure 3 shows a typical schematic structure of a marine power plant. A marine power plant generally consists of various macinery and equipment. In the figure, notations of symbols express the main engine

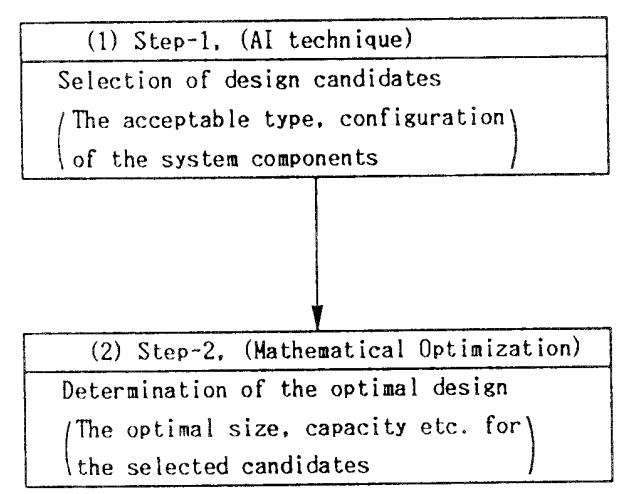

Fig. 2 Concept of the two-level expert system coupling the AI technique and the mathematical optimiza. tion 


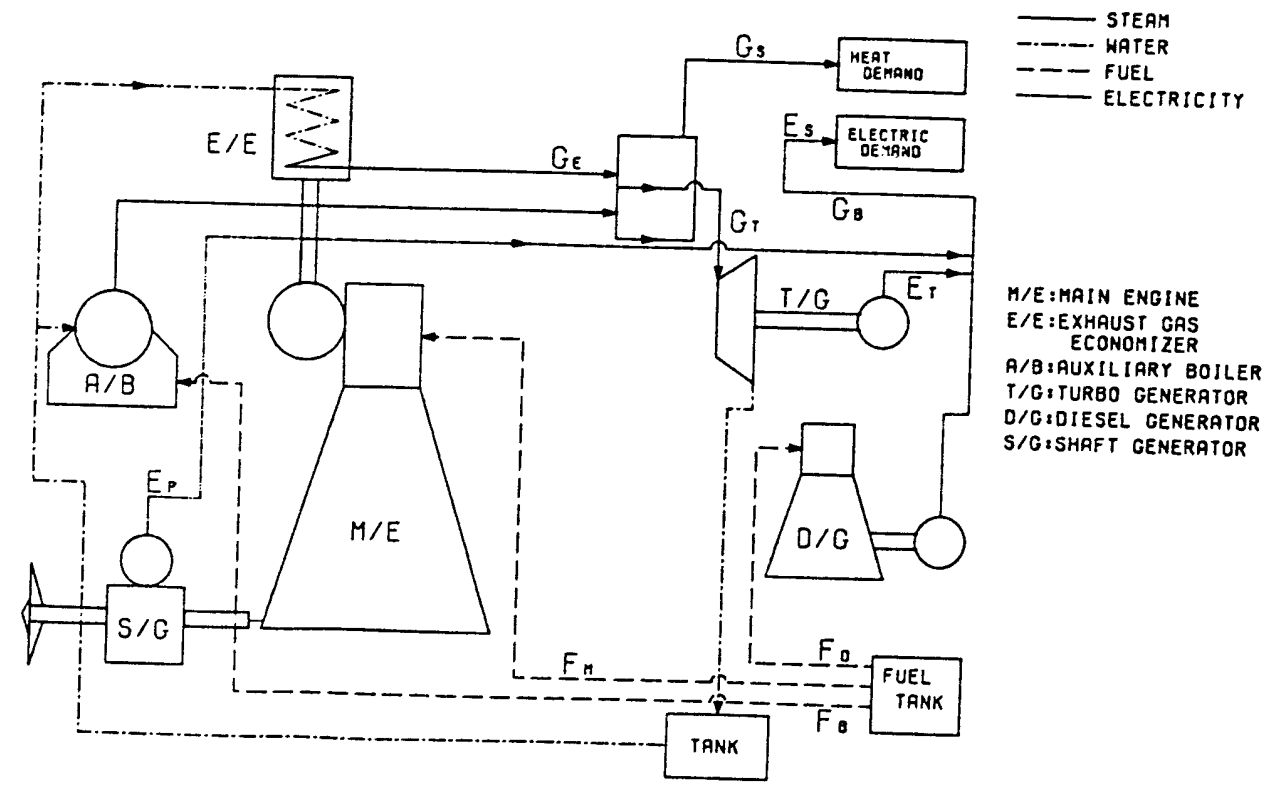

Fig. 3 Typical schematic structure of a marine power plant

$(\mathrm{M} / \mathrm{E})$, the diesel generator $(\mathrm{D} / \mathrm{G})$, the turbogenerator $(T / G)$, the shaft generator $(S / G)$, the auxiliary boiler $(\mathrm{A} / \mathrm{B})$ and the exhaust gas economizer $(E / E)$, respectively. The main engine $(M / E)$ is connected to a propulsion shaft which drives a propeller.

The above machinery and equipment other than the main engine $(\mathrm{M} / \mathrm{E})$ constitute a heat and electric power generating plant which supplies energy for various purposes for the ship's use: i. e., hot water supply, heating fuels, lighting, air-conditioning, refrigeration, and so forth.

It is a problem in the initial design of a marine power plant ;

(1) to select and determine the acceptable type and size of the "main engine" considering the kind of ship, the ship's size, speed, and so forth, so as to satisfy the various design conditions, including optimal operational economy.

(2) to select and determine the acceptable type and size of "machinery and equipment" so as to satisfy the heat and electric power demand. In the plant, however, there exist many alternative combinations among the type and size of machinery and equipment. Therefore, in the design process of the plant, it is important to determine their rational system configuration and operational condition to minimize the operating cost, including the fuel cost for various energy demand patterns in the ship's voyage conditions.

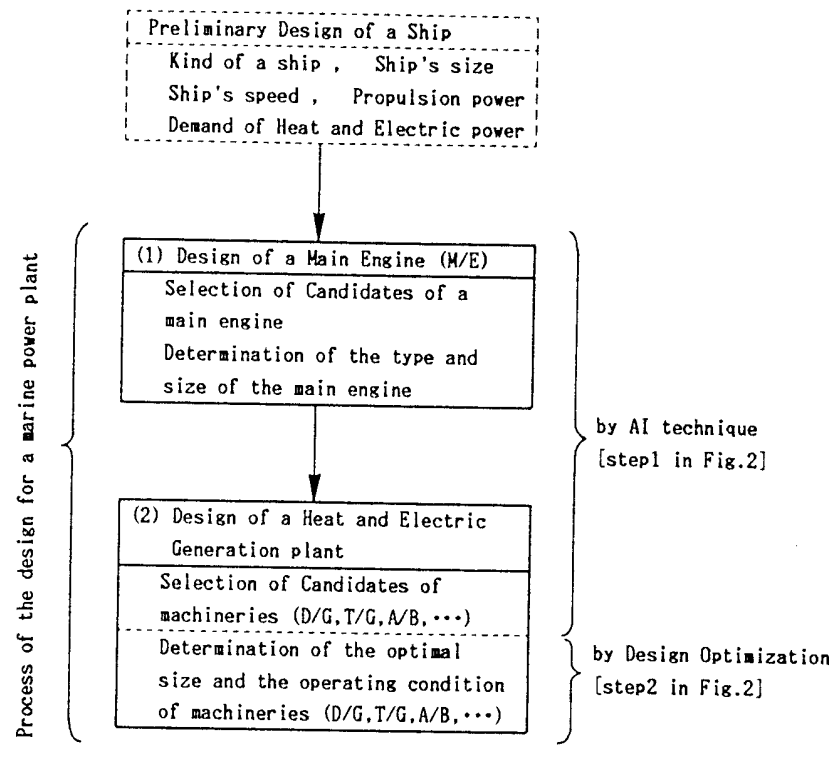

Fig. 4 Process of the design for a marine power plant

- This design process is shown in Fig. 4.

In Fig. 4, the design condition for the plant is given by the result of the preliminary design of a 'ship' which includes the kind of ship, the ship's size, speed, propulsion power, and the demand for heat and electric power. The details of the ship design itself are not discussed here, but the results are assumed to be a given design condition for the ship's plant - the marine power plant - . After it is given, the design process of the marine power plant begins :

Firstly, the type and size of the main engine is 
determined as mentioned in the above item (1). Then, the heat and electric power generating plant is determined as mentioned in the item (2) above.

\section{The Expect CAD System for the Design of a Marine Power Plant}

The design process explained in chapter 4 can be implemented actually as an expert system by combining the AI technique and the optimization method.

\section{1 System construction and knowledge repre. sentation}

Figure 5 shows an implemented system. The system consists of ;

(1) The inference engine, to control the design process,

(2) The process memory, to watch the design process,

(3) The knowledge base, to include the knowledge elements for the design,

(4) The database, to include the data of various types of main engines and machinery constituting the plants,

(5) The design optimization, to optimize the design variables of the candidates,

(6) The optimization computer programmes, for numerical optimization.

It is important for constructing an expert system what type of knowledge base should be adopted to represent the engineering design knowledge. There are several well-known types of knowledge representation $^{(6) \sim(8)}$.

In this investigation however, no detailed explanation is made for the type of knowledge representation, but "Production rules" and "Frame based representation" will be adopted for the system.

The design process control (1) determines which "rules" are to be applied in the process while design is proceeding. Then, the applied rules are described in the process memory (2) one by one. The knowledge implemented in the design process control is comprised of so-called "meta-rules"(6) (8), which is the knowledge needed to control the following "ruled knowledge elements".

The design knowledge (3) in Fig. 5 represents the heuristic knowledge which is generally acquired from experienced design experts. They are usually described with "production rules", which take the form; "If $A$, then $B$ ". If the given condition matches " $A$ ", then the action of " $B$ " is taken. The action is to manipulate knowledge, for example, to search the candidates of a main engine in the database or to perform a calculation procedure.

The database (4) in Fig. 5 includes the standard data of various main engines or machinery which are described as the "frame-based representation". They are of originally "catalogued-data" including the machineries' characteristics, dimensions, and so forth. When the candidates are selected from the standard frame data in the database, they are constructed as the specific data of the plant and are described in the same database.

Then, the design optimization procedure (5) in Fig. 5 is carried out using a mathematical programming method. The objective functions are usually selected to minimize the total cost of the plant.

The computer languages used are LISP ${ }^{(9)}$ for the AI procedure and FORTRAN for the mathematical programming respectively.

\section{2 Examples of knowledge representation and its handling}

Examples of the knowledge representation of the expert system for a marine power plant design are explained.

(1) Production rules for the design knowledge:

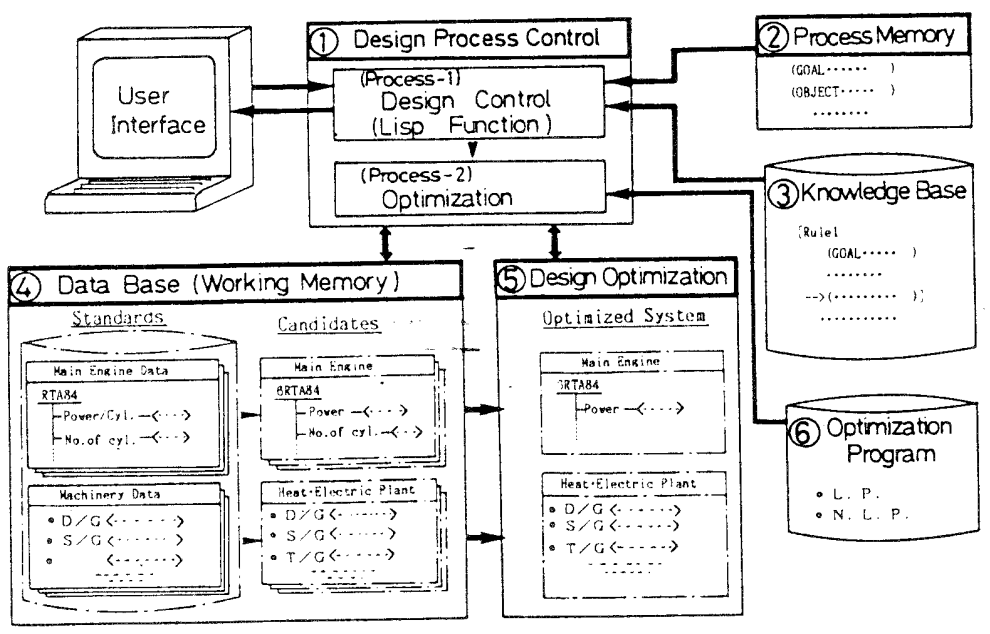

Fig. 5 Expert system for the marine power plant design 
The respective design knowledge is represented by the production rules. Some examples are as follows ;

\# "If the kind of ship is a tanker or a LNG carrier ship, and the required power of the ship is greater than $55000 \mathrm{PS}$, then the main engine is to be a turbine, and all else is to be diesel".

\# "If the kind of ship is a ferry-boat, then the main engine is to be a medium-speed diesel, and all else is to be low-speed diesel."

\# "If the power of the main engine is lower than $55000 \mathrm{PS}$, then the number of the engines is to be 1 -set, and all else is to be 2 -sets.

These rules are represented in symbolic forms and are programmed in LISP language as shown in Fig. 6 .

(2) Frame representation for the machineries' data:

The engineering data for the machineries are described in the frame-based representation. The examples for a main engine are shown in Fig. 7. The frame consists of the engine's type, the size of the cylinder, the revolution, the main dimensions and so forth. These data are connected to each other with the 'slot'(9) value. By means of the slot representation, the items described in the candidate data are inherited from the basic items described in the standard data; which means that the common items described in the standard data can be automatically referred to the candidates data.

( 3 ) An example of knowledge handling:

An example shows how the design knowledge and the frame data are handled in the system. The example is taken for the procedures in which the candidate of the main engine is selected from the standard main engines' data

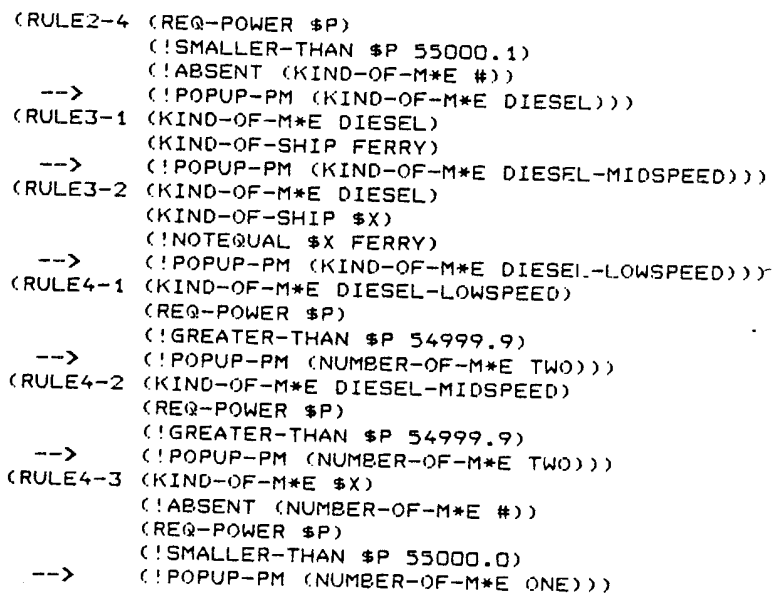

Fig. 6 Examples of rules in LISP language listed in the data-base. These procedures are conducted by the various rules described in the knowledge base.

To explain the procedures, two-diagrams Fig. 8 (a) and (b) are shown:

(a) The power range diagram of the main engine shown as Fig. 8(a), to select the type of the engine,

(b) The specific fuel consumption shown as Fig. 8 (b) which defines the fuel consumption corresponding to the power range of Fig. 8(a).

The power range diagram, Fig. 8 (a), shows the layout area within which there is freedom to select the combination of the power output $(\mathrm{kW})$ and speed ( $\mathrm{r}$. p.m. ) for the candidates of the engines, which satisfy the requirement of the ship's operating condition. Then, the specific fuel consumption, Fig. $8(\mathrm{~b})$, is determined for the respective candidates of the engines.

In these procedures, several candidates for engines are found within the required range of the output and the speed, and then respective fuel consumptions are given in Fig. 8( b ). Finally, the engine is determined from among them so that it given the minimum total operating cost (sum of the fuel cost and the initial cost).

\section{3 Optimization Process}

(1) Formulation of optimization modeling After

Slandard Data (Main Engine)

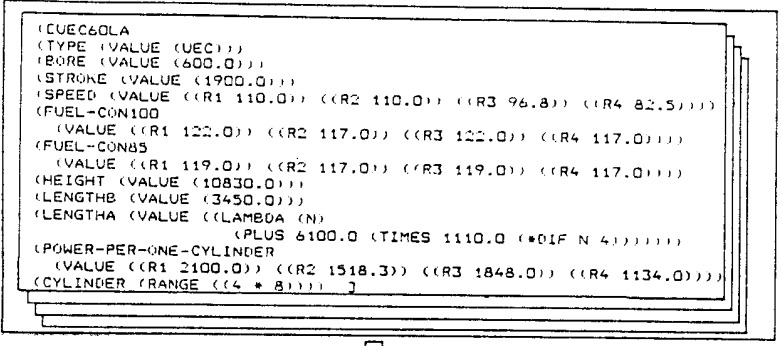

Candidate Data (Main Engine)

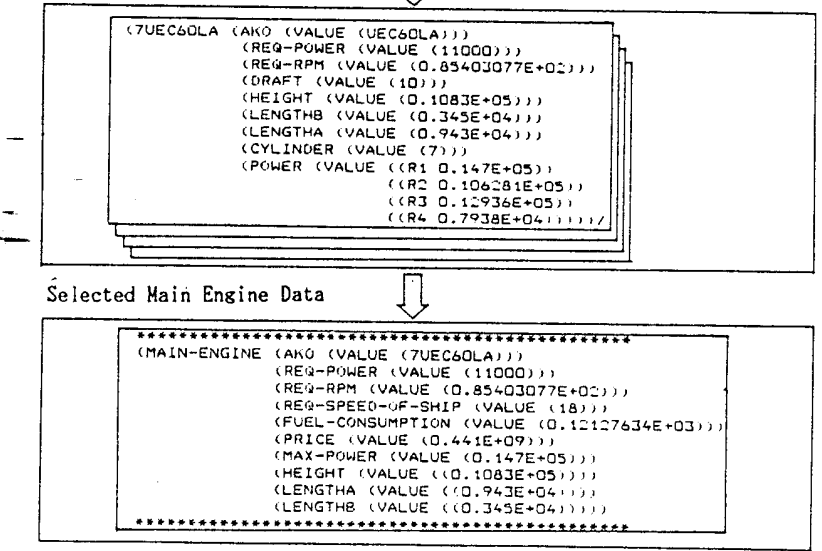

Fig. 7 Data of the main engine (Frame representation) 
several candidates of machinery for the plant are selected by the AI technique, the design optimization procedure is then adopted to squeeze them by using a mathematical programming method, as mentioned in 5.1. The design objective is taken to be to minimize the annual operating cost which consists of the fuel cost and the initial cost of the plant.

However, the fuel cost varies due to the ship's operating conditions, i. e., the ship's route, speed and loading condition, as well as to long-term voyaging conditions which are determined by the annual operating days. This means that the working point of the machinery of the plant is complexly affected by the variation of the thermal and electric damand given by the ship's operating condition. The optimal operating point can be determined by the mathematical optimization method so as to minimize the annual fuel cost for the combinations of the given candidates of the machineries and the equipment constituting the plant. Then, the optimal construction of the plant is determined from the result of the optimization.

The above optimization problem is formulated as follows:

The objective function is selected so as to minimize the annual fuel cost $Z$, namely,

$$
\begin{aligned}
& \min . Z=\sum_{m}\left\{\varphi_{A} \sum_{i} F_{A_{i}}^{(m)}\right. \\
& \left.\quad+\varphi_{C}\left(\sum_{j} F_{C_{j}^{(m)}}^{(m)}+\sum_{k} F_{c k}^{(m)}\right)\right\} T^{(m)}
\end{aligned}
$$

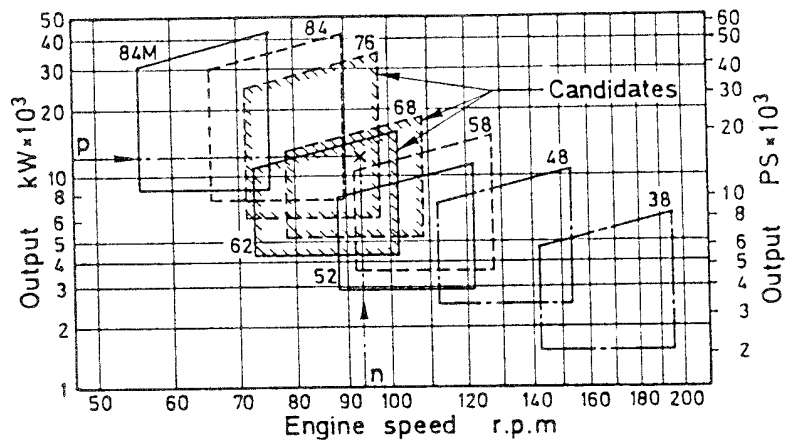

(a) Power Range

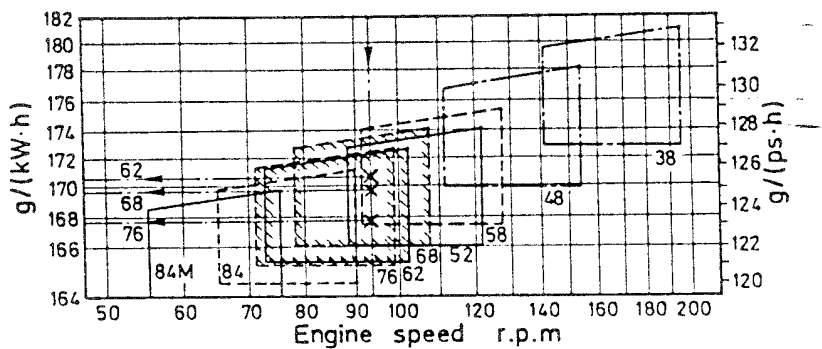

(b) Specific Fuel Consumption

Fig. 8 An example of knowledge handling (Selection algorithm of the main engine) where,

$\varphi_{A}, \varphi_{C}$ : prices of light and marine fuel oils respectively,

$F_{A i}$ : fuel consumption by the $i$-th diesel generator $(0 \leq j \leq 3)$,

$F_{c j}$ : fuel consumption by the $j$-th auxiliary boiler $(0 \leq j \leq 3)$,

$F_{C k}$ : fuel consumption by the $k$-th shaft generator $(0 \leq k \leq 3)$,

$T$ : annual voyaging hours,

Superscript $(m): m$-th voyage pattern.

Constraints are expressed by the equations which describe the relations among the design variables, they are:

(i) The performance curves of the machinery representing the power relations as shown by Fig. 9 ,

(ii) The flow balance relations as shown by Fig. 3, namely,

(a) Enthalpy flow balance of steam,

(b) Mass flow balance of fuel,

(c) Electric energy flow balance.

Under the above constraints, the performance curves include the On-Off condition of the machinery, as illustrated in Fig. 9. This condition can be expressed with $0-1$ integer variables. The optimization problem, including these kind of variables is formulated as mixed-integer programming (10),(11).

(2) Solution of the optimization problem Some mathematical solving algorithms are proposed for the linear case $\mathrm{s}^{(10) \sim(12)}$. As the above problem is formulated by linear functions, the solutions can be obtained for the optimal operating points

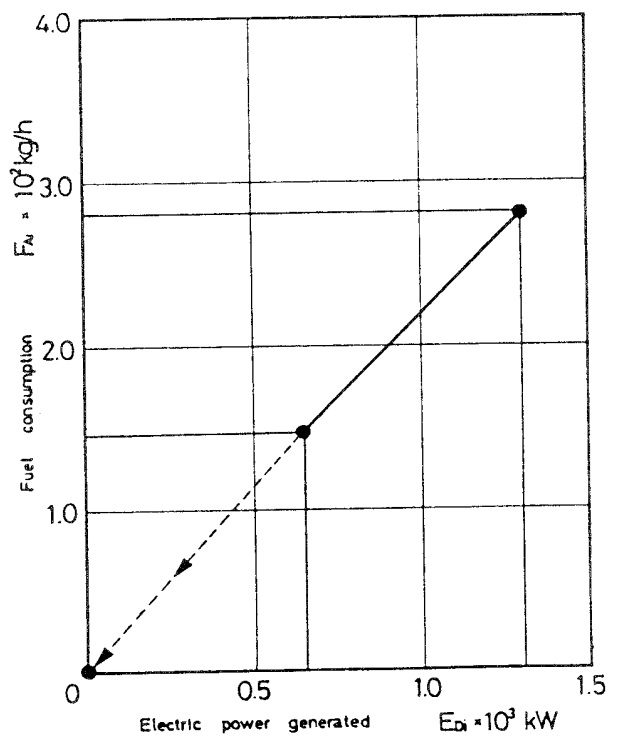

Fig. 9 An example of the performance curve (D/G) 
$F_{A i}^{*(m)}, F_{C j}^{*(m)}$ and $F_{C k}^{*(m)}$ for the $m$-th voyage pattern, so as to minimize the fuel cost $Z$. Then, the annual total operating cost (TC) can be given by adding the following annual fixed cost $(\mathrm{C})$.

$$
C=\gamma P
$$

where, $P$ : price of the plant, $\gamma:$ capital recovery factor, as given by equation $(3)$.

$$
\gamma=\rho(1+\rho)^{N} /\left\{(1+\rho)^{N}-1\right\}
$$

where, $\rho$ : the annual interest rate, $N$ : number of interest periods in year.

The optimization problem is finally given as,

Min. $T C=C+\operatorname{Min} . Z$

where, Min. $Z$ is given by equation ( 1 ).

The solution procedure for the numerical optimization is programmed in FORTRAN which can be automatically recalled from the main programme with LISP language.

\section{A typical Trace of the System Execution}

The developed expert system was implemented on a large computer system (NEC ACOS 1000 system of the Osaka University Computation Center) which was able to access from personal computer terminals.

For the purpose of ascertaining the validity and effectiveness of the developed expert system, an example of the system execution is presented here for the design of the power plant of a containership.

The system execution proceeds interactively. In Fig. 10, a brief explanation is given for a typical design process of selecting the main engine.

Mark (1): The design conditions are given for the kind of ship, the ship's speed (knot), draft $(m)$ and required power MCR (PS).

(2): ' 9 candidates' of the main engine are selected based on the design knowledge implemented in the system.

(3): Candidates are evaluated from the following three view points;

1) 3 candidates are recommended in the order of lower 'fuel consumption'.

2) 3 Candidates are recommended in the order of lower 'initial cost'.

3) 3 candidates are recommended in the order of lower 'total cost'.

Then, the main engine is finally determined by a designer.

(4): The configuration of the selected main engine is displayed.

In Fig. 11, a brief explanation is given for a typical design process for determining the heat and electric power generation plant.

Mark (1): The design conditions are given by the electric demands and the heat demands.

(2): Kinds of machineries and equipment composing the plant are indicated.

(3) : 108-Combinations of machineries and equipment are given to the candidates of the plants.

(4): Calculation for the optimization is performed. As a result of the optimization, the best 10 candidates of the plant are shown from the view point of initial cost. And also, the best 10 candidates of the plant are shown from the view point of total cost, including fuel cost. The No. 106 plant, table 106, is indicated as the optimal. The final decision to select the No. 106 plant is made by a designer.

(5): The layout of the No. 106 plant is displayed, together with the list of particulars of machinery composing the plant.

Although the above example is but a limited part of the procedure, the effectiveness of the system is clearly demonstrated.

\section{Conclusions}

Engineering design is considered as an ill-

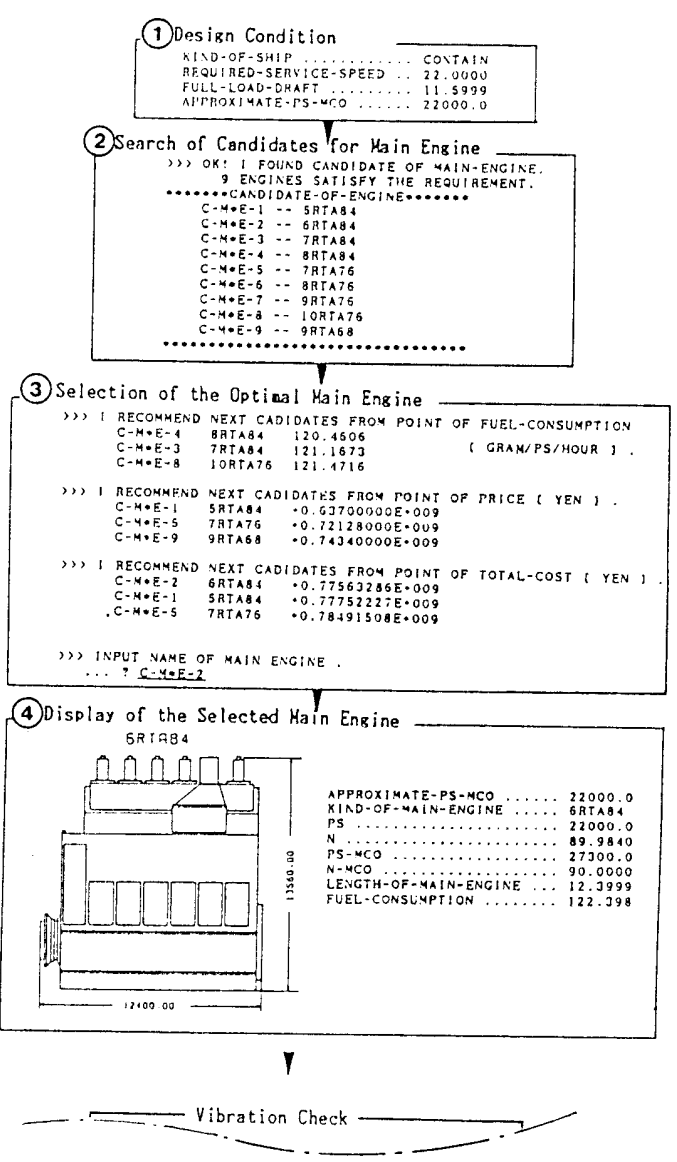

Fig. 10 Typical design process of selecting the main engine 


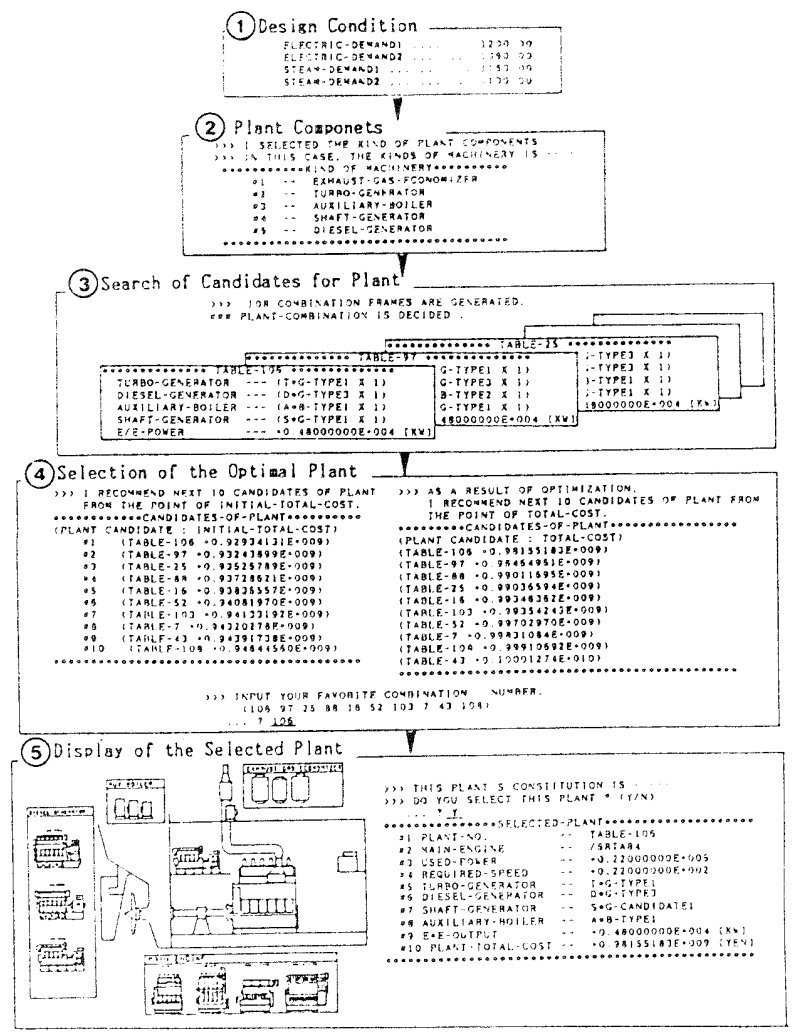

Fig. 11 Typical design process of determinating the heat and electric power generation plant

structured process which lacks well-defined goals and solving algorithms. The AI technique, especially expert systems, provides promising tools which expand computer applications to engineering design. An investigation was done to introduce the AI technique effectively to engineering design. The results obtained are summarized as follows:

(1) Discussion to understand the engineering design process from the view point of applying the AI technique to the design was made. It is ascertained that the design process can be divided into 4 -steps, namely, '(1) Recognition of design needs', '(2) Search of the design candidates', '(3) Evaluation of the acceptable design solution' and '(4) Design optimization'. The AI technique is especially effective to the design process for (2) and (3) steps.

(2) Based on the above understanding, an expert CAD system coupling the AI technique for (2) and (3) steps and the numerical optimization procedure for step (4) was proposed to support the wide domain of the design process.

(3) An expert system was developed for the basic design of an actual marine power plant in order to ascertain the validity and effectiveness of the above proposal.
(4) In the system, the design knowledge was represented in 'production rules', and the various engineering data of the machinery composing the plant were described in a database using the 'frametype representation'. The system construction is a hybrid-type combining the $\mathrm{AI}$ and the numerical optimization based on the above result (2).

(5) The system thus developed was found to be effective not only as a tool for assisting designers but also as a tool for instructing inexperienced designers.

\section{Acknowledgements}

The authors wish to thank Mr. K. Shimamoto and Mr. R. Enomoto of Mitsubishi Heavy Industries for valuable help with acquiring knowledg about marine power plant design.

\section{References}

(1) Dixon, J. R. and Simmons, M. K., Computers That Design, (Expert Systems for Mechanical Engineers), ASME Computers in Mech. Eng., (198311), p. 10 .

(2) Applications of Knowledge-Based Systems to Engineering Analysis and Design, (ed. Dyn, C. L. ), ASME, WAM, (1985-11).

(3) Knowledge Engineering in Computer-AidedDesign, (ed. Gero, J. S. ), Proc. of IEIP WG 5.2, North-Holland, Amsterdam, (1984-9), Elsevier Sci. Pub.

(4) Applications of Artificial Intelligence in Engineering Problems, (eds. Sriram, D. and Adey, R.), Vol. I, II, Ist Int. Conf., New York, (1986-4), Springer-Verlag.

(5) Malone, D. W., An Introduction to the Application of Interactive Structural Modeling, Proc. IEEE, Vol. 63, No. 3(1975-3), p. 307.

(6) Barr, A. and Feigenbaum, E. A., The Handbook of Artificial Intelligence, Kaufmann, Los Altos, Calif., (1981).

( 7 ) Hayes-Roth, F., Waterman, D. A. and Lenat, D. B., Building Expert System, Reading, Mass., (1983), Addison-Wesly.

(8) Harmon, P. and King, D., Expert Systems, Reading, Mass., (1985), Addsion-Wesley.

(9) Winston, P. H. and Horn, B. K. P., LISP, Reading, Mass., (1981), Addsion-Wesley.

(10) Garfinkel, R. S. and Nemhauser, G. L., Integer Programming, (1972), John Wiley \& Sons, New York.

(11) Pulleyblank, W. R., Progress in Combinational Optimization, (1984), Academic Press, New York.

(12) Akagi, S., Yokoyama, Y., and Ito, K., Trans. Jpn. Soc. Mech. Eng., (in Japanese), Vol. 52, No. 476, C (1986), p. 1469. 